\title{
Successful Treatment of Extranodal Natural Killer T Cell Lymphoma Nasal Type Complicated by Severe Hemophagocytic Syndrome A Case Report
}

\author{
Wei Shui ${ }^{1}$, Qianying Pan ${ }^{2}$, Yuanyuan Niu ${ }^{1}$, Yanan Liu ${ }^{3}$ and Changran Zhang ${ }^{1 *}$ \\ ${ }^{1}$ Department of General Practice, The East Division of the First Affiliated Hospital of Sun Yat-sen University, Guangzhou, \\ Guangdong, China
}

${ }^{2}$ Department of Hematology, The First Affiliated Hospital of Sun Yat-sen University, Guangzhou, Guangdong, China

${ }^{3}$ Department of Hematology, The First Affiliated Hospital of Guangdong Pharmaceutical University, Guangzhou, Guangdong, China

*Corresponding author: Changran Zhang, Department of General Practice, The East Division of the First Affiliated Hospital of Sun

Yat-sen University, Guangzhou, Guangdong, China

\section{ARTICLE INFO}

Received: 蔧 September 02, 2021

Published: 慧 September 15, 2021

Citation: Wei Shui, Qianying Pan, Yuanyuan Niu, Yanan Liu, Changran Zhang. Successful Treatment of Extranodal Natural Killer T Cell Lymphoma Nasal Type Complicated by Severe Hemophagocytic Syndrome A Case Report. Biomed J Sci \& Tech Res 38(5)-2021. BJSTR. MS.ID.006202.

Keywords: Extranodal Natural Killer/Tcell Lymphoma; Hemophagocytic Syndrome; Severe Pneumonia; Chemotherapy; Immunotherapy

\begin{abstract}
Extranodal natural killer/T-cell lymphoma, nasal type (ENKL) is a subtype of mature T-and NK-cell lymphomas characterized by its association with Epstein-Barr virus and extranodal involvement. It is frequently complicated with HPS, and prognosis is poorer in this situation. A correct diagnosis usually requires the histopathological examination. Here we report a case of 32-year-old man presented with a month-long high-grade fever, cough, chest pain and blood in phlegm. A chest computed tomography scan detected pneumonia-like features, including multiple variable nodules, groundglass opacities and patchy infiltration, which progressed rapidly. The patient was initially diagnosed with severe pneumonia and received a combination treatment of broad-spectrum antibiotics , however the patient's condition continued to deteriorate. Biopsy of lung and soft tissue confirmed a diagnosis of extranodal NK/T-cell lymphoma, nasal type. Bone marrow aspiration did not show lymphoma involvement. The patient had progressive neutropenia upon presentation, with further investigations showing hepatosplenomegaly, hyperferritinemia, hypertriglyceridemia, low natural killer (NK) cell activity and increased circulating soluble IL-2 receptor. We reached a diagnosis of hemophagocytic syndrome. Chemotherapy and immunotherapy were started leading to a progressive improvement of the disease.
\end{abstract}

Abbreviations: ENKL: Extranodal Natural Killer/T-Cell Lymphoma; EBV: Epstein-Barr Virus; UADT: Upper Aerodigestive Tract; HLH: Hemophagocytic Lymphohistiocytosis; HPS: Hemophagocytic Syndrome; LAHS: Lymphoma-Associated Hemophagocytic Syndrome

\section{Introduction}

Extranodal Natural Killer/T-Cell Lymphoma, Nasal Type(ENKL), is a rare malignancy of Non-Hodgkin lymphoma characterized by an aggressive clinical course and poor prognosis [1]. It is mostly endemic to East Asia and is associated with Epstein-Barr Virus (EBV) infection [2]. Its lesions are predominantly present in the Upper Aerodigestive Tract (UADT) such as the nasal cavity, nasopharynx, paranasal sinuses or palate. Less commonly, ENKL can manifest at extra nasal locations like the lung, skin, soft tissue, gastrointestinal tract, and testis. Since the neoplasm can destroy the midline facial structures, the disease used to be known as lethal midline granuloma $[3,4]$.The presentation of the disease at extra nasal locations is nonspecific and may mimic many other benign or malignant lesions. Patients with extra nasal presentation often have 
more adverse clinical features such as an advanced stage, elevated LDH and poor performance status, and the survival rate is inferior compared with the nasal sites [5-7]. Hemophagocytic Syndrome (HPS), also known as Hemophagocytic Lymphohistiocytosis (HLH), is highly heterogeneous and comprises primary and secondary types.

Secondary HPS is associated with a variety of underlying conditions such as infection, malignancy, and autoimmune diseases. ENKL is frequently complicated with HPS, and survival is discouraging in this circumstance [8]. However, no standard treatment has been established based on the results of randomized controlled trials because of the rarity of the disease [9]. Here, we report a case of ENKL complicated by severe LymphomaAssociated Hemophagocytic Syndrome (LAHS). The patient was treated according to the R-DEP (ruxolitinib, liposomal doxorubicin,VP-16, dexamethasone) chemotherapeutic regimen, and the hemophagocytic lymphohistocytosis gradually improved during chemotherapy. Then P-Gemox (pegaspargase, gemcitabine, oxaliplatin) chemotherapy in combination with the use of antiPD1 antibody (Sintilimab Injection ) were performed and achieved PR(partial remission). For personal reasons, the patient chose a different hospital to continue the treatment. He achieved CR (Complete Remission) with the therapy of anti-PD1 antibody and Chidamide. Unit now the patient has received the therapy for almost one year and follow up regularly in outpatient department.

\section{Case Report}

A 32-year-old non-smoking man was emergently transferred to our hospital on June 30,2019 , because of a month-long highgrade fever, cough, left-sided chest pain and blood in phlegm. Prior to this, the patient had a history of trauma to the right lower limb and the wound healed itself. About ten days later he presented to a local hospital with the above-mentioned chief complaint and a chest computed tomography scan showed multiple nodules, ground-glass opacities and patchy infiltration scattered in both lung fields. Then he had been treated as for pneumonia. However, his clinical condition did not improve. The patient was therefore admitted to our hospital for further evaluation and treatment. There was no relevant personal or family medical history for this patient. The physical examination upon admission revealed a palpable mass $(3 \mathrm{~cm})$ on the left dorsal side and the lower margin of the right 9th rib, respectively. Fine rales were heard in the lower left lung. Hematologic examination showed a white blood cell count of $2.67 \times 10^{9} / \mathrm{L}$, a hemoglobin concentration of $11.6 \mathrm{~g} /$ $\mathrm{dL}$, and a platelet count of $77 \times 10^{9} / \mathrm{L}$. Serum chemistry showed abnormal results as follows: aspartate aminotransferase (AST) 115 IU/L, alanine aminotransferase (ALT)133 IU/L, fibrinogen 1.13g/L, lactate dehydrogenase 966IU/L (normally 114-240 IU/L),Creactive protein $25.53 \mathrm{mg} / \mathrm{L}$ (normally less than $10 \mathrm{mg} / \mathrm{L}$ ), the procalcitonin level $0.17 \mathrm{ng} / \mathrm{ml}$ (normally $<0.05 \mathrm{ng} / \mathrm{ml}$ ), triglyceride $2.96 \mathrm{mmol} / \mathrm{L}$ (normally $0.33-1.7 \mathrm{mmmol} / \mathrm{L}$ ), serum ferritin8141.3 $\mu \mathrm{g} / \mathrm{L}$ (normally 20-200 $\mu \mathrm{g} / \mathrm{L}$ ), soluble interleukin (IL)- 2 receptor $>7500 \mathrm{U} / \mathrm{mL}$ (normally $223-710 \mathrm{U} / \mathrm{mL}$ ) and natural killer (NK) cell activity $1.75 \%$.In addition, his plasma EBV DNA level was 4300 copies $/ \mathrm{ml}$. A bone marrow aspiration did not show lymphoma involvement (Figure 1).

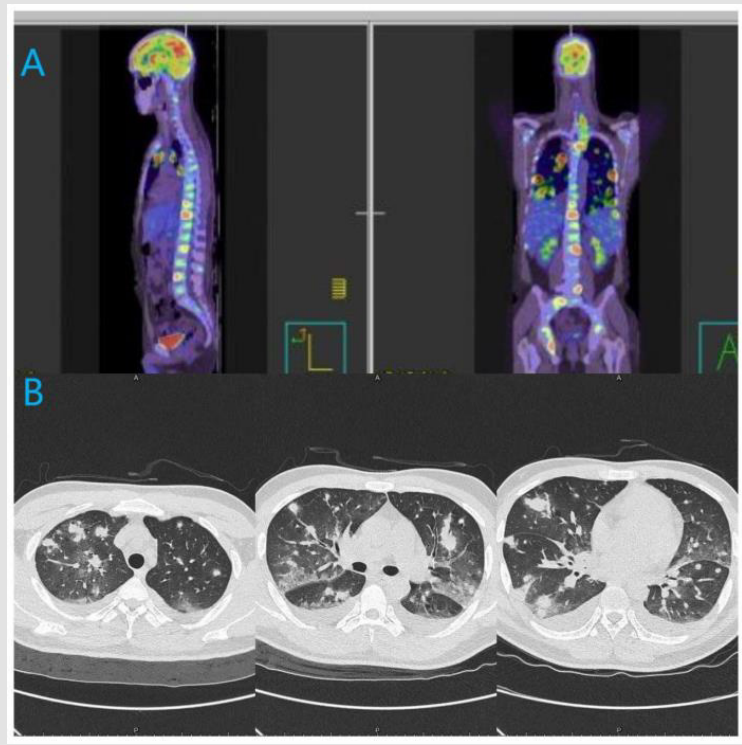

Figure 1:

a) PET/CT revealing heterogeneous hypermetabolic masses in both lung fields, as well as hepatic, splenic, osseous, soft tissue, and multiple lymph node metastases that involved the mediastinum, porta hepatis, bilateral hilum of lung.

b) Chest CT scan showing multiple nodules, ground-glass opacities and patchy infiltration in both lungs. 
The patient was HIV negative. Two sets of blood cultures, and tumor markers, including CEA,SCC, CFRA21-1,Pro-GRP, and NSE were all normal. The G test and GM test were both negative. Antinuclear antibody, anti-ENA antibodies, and anti-neutrophil cytoplasmic antibodies were all negative. Administration of broadspectrum antibiotics did not resolve hissymptoms. Bronchoscopic examination did not give a definite diagnosis. Lung nodules increased and grew larger, and hypoxia progressed. A CT-guided transthoracic needle biopsy of the left lower lung was performed (Figure 2). During the course of treatment, FDG PET/CT was conducted and revealed heterogeneous hypermetabolic masses in both lung fields, as well as hepatic, splenic, osseous, soft tissue on the left dorsal side and the lower margin of the right 9th rib, and multiple lymph node metastases that involved the mediastinum, porta hepatis, bilateral hilum of lung. Then we also performed a ultrasound-guided percutaneous puncture biopsy of soft tissue at the lower margin of the right $9^{\text {th }}$ rib. Immunohistochemical staining of these two specimens both yielded positive results for CD56, CD3, CD2, and the Ki-67 proliferation index was 80\%;in situ hybridization for EBV-encoded early small RNAs (EBER) was also positive; however, results were negative for CD79a,CD20.
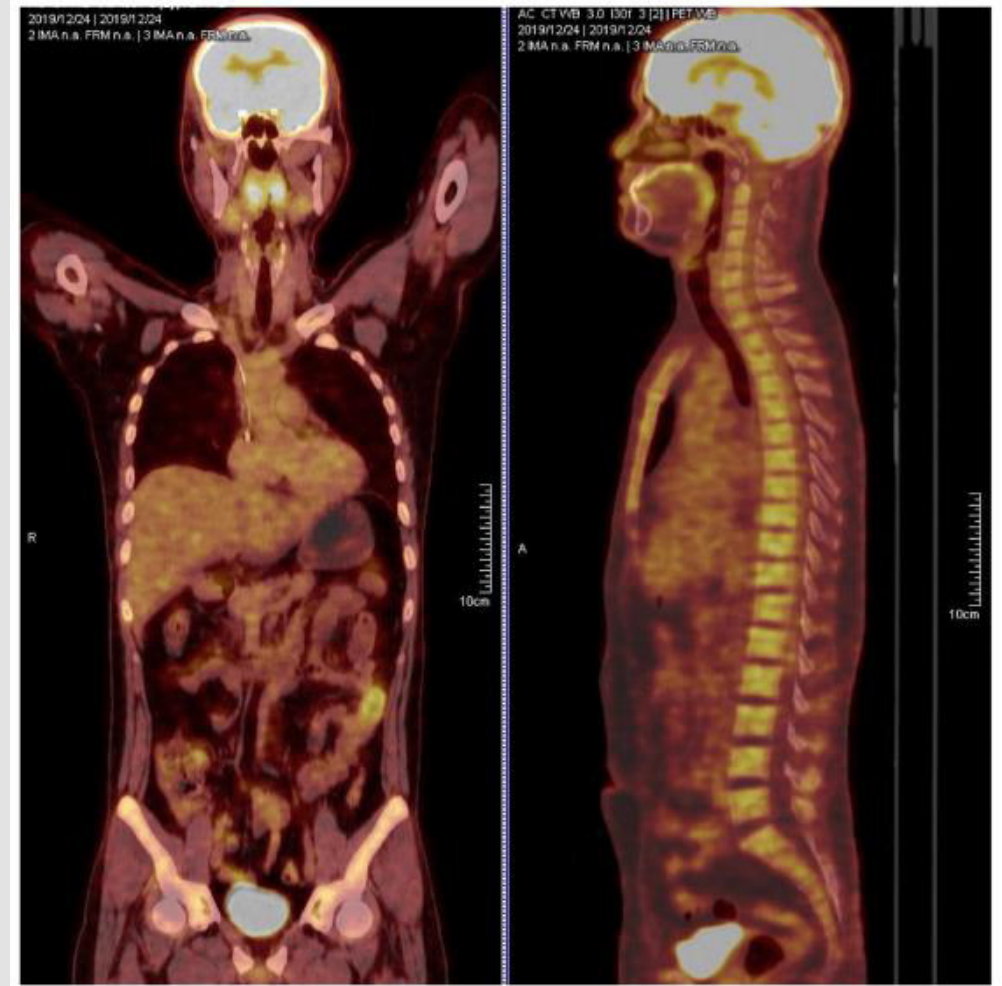

Figure 2: PET-CT on December 24, 2019, showing that the lesions almost disappeared.

Moreover, Hemophagocytic lymphohistiocytosis was confirmed by cytopenia, fever, splenomegaly, hyperserotonemia, hypertriglyceridemia, low natural killer (NK) cell activity and increased circulating soluble IL-2 receptor. A diagnosis of ENKL complicated by LAHS was made. The markedly elevated circulating plasma EBV-DNA level supported the diagnosis. R-DEP(ruxolitinib, liposomal doxorubicin,VP-16,dexamethasone) chemotherapeutic regimen was performed to control the LAHS and it gradually improved during chemotherapy. Then six cycles of P-GEMOX(pegaspargase, gemcitabine, oxaliplatin) chemotherapy in combination with the use of anti-PD1 antibody(Sintilimab
Injection) were performed and achieved PR(partial remission) after the treatment. Although the patient suffered from septic shock caused by Klebsiella pneumoniae in the neutropenic period, he was cured by broad-spectrum antibiotics when neutrophil improved. For personal reasons, the patient chose a different hospital to continue the treatment. He achieved CR(complete remission) confirmed by using positron emission tomography-computed tomography (PETCT)with the therapy of anti-PD1 antibody(Tislelizumab Injection) and Chidamide. Until now the patient has received the therapy for more than one year and follow up regularly in outpatient department (Figure 3). 


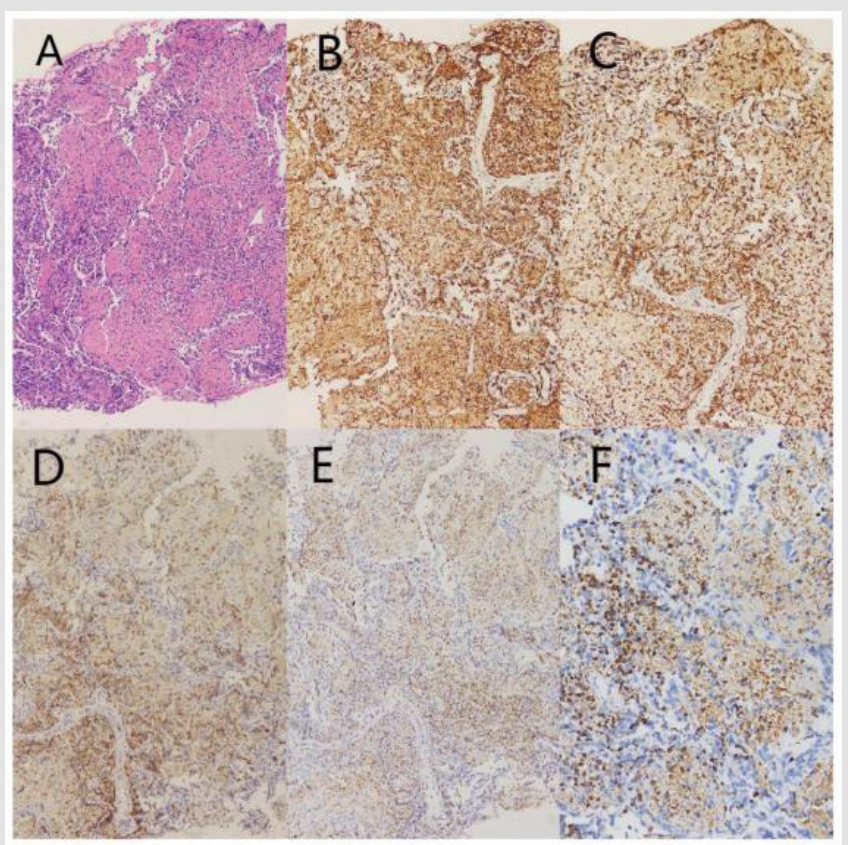

Figure 3: Pathologic findings in CT-guided transthoracic needle biopsy specimens.

a) Histologically, a small number of nuclear hyperchromatic cells presented with large areas of observable necrosis(HEstaining;magnification, $\times 400$ ).

b) Immunohistochemical staining positive for the expression of CD2(magnification, $\times 400$ ).

c) Immunohistochemical staining positive for CD3 expression (magnification, $\times 400$ ).

d) Immunohistochemical staining positive for the expression of CD56 (magnification, $\times 400$ ).

e) Immunohistochemical staining positive for the expression of TIA-1(magnification, $\times 400$ ).

f) In situ hybridization positive for EBV-encoded RNA (magnification, $\times 400$ ).

\section{Discussion}

To our knowledge, there have been only a few reports documenting long-term remission in patients with ENKL complicated by severe LAHS. Han et al conducted a study comparing NK/T-LAHS with LAHS associated with other T cell lymphomas, which indicated that both had poor prognosis with a median survival time of 28 and 33 days, respectively [10]. Chang, et al. [11] identified that a long diagnosis time was a poor prognostic factor for patients with LAHS. So the early identification of lymphoma-associated HPS is essential to improve patient, prognosis. However it is challenging since misdiagnosis often occurs as fever and pancytopenia may also be caused by severe infection. The CT findings of pulmonary non-Hodgkin's lymphoma are varied and nonspecific. Patchy consolidations, nodules, and masses are the most frequent CT findings and infiltrations can also be seen [12]. It is reported that PET-CT may act as a significant tool to assess patients with LAHS, as it is highly sensitive in detecting neoplasms of the majority of histologic subtypes of lymphoma, and also demonstrates extensive 18-fluorodeoxyglucose (FDG) uptake in tumor tissues [13]. Anyhow there is no non-invasive test specific enough to make a correct diagnosis of ENKL. So lymphoma complicated with HPS should be considered in patients presenting with fever, cough, dyspnea and pancytopenia, associated with unilateral or bilateral pulmonary consolidation and pleural effusion, when combination therapy involving numerous antimicrobial agents has failed. A proper diagnosis may be established by a histopathological examination.

The International Peripheral T-cell Lymphoma Project demonstrated that extranasal NK/T-cell lymphoma (nasal type) has worse clinical features and survival rate, even in cases with apparently localised disease, than nasal NK/T-cell lymphoma in extranodal NK/T cell lymphoma [14].In this report, the tumor involved multiple organs throughout the patient's body. As with the progressive disease courses and poor prognosis, effective therapeutic strategies are urgently needed. As for HPS, the patient was treated according to the R-DEP(ruxolitinib, liposomal doxorubicin,VP-16,dexamethasone) chemotherapeutic regimen, and the overall condition of the patient gradually improved during chemotherapy. Nevertheless, it was equally important to treat primary diseases as well as treating HPS [11]. Regarding ENKL, no standard treatment has been established based on the results of randomized controlled trials because of the rarity of the disease [9]. ENKL cells are associated with a high expression of P-glycoprotein, 
leading to multidrug resistance that is likely responsible for the poor response to conventional anthracycline-based chemotherapy [15]. P-GEMOX is a modification of the Gemcitabine, L-Asparaginase, and Oxaliplatin (GELOX) regimen in which L-asparaginase is switched to pegaspargase and was also included as a suggested treatment regimen for ENKL in the NCCN guidelines [16]. In a retrospective analysis of 117 patients with ENKL (96 with newly diagnosed ENKL and 21 with relapsed/refractory(R/R) disease), the P-GEMOX regimen resulted in an ORR of $88 \%$ and responses were similar for patients with newly diagnosed and R/R ENKL [17].

Recently, newer agents for ENKL, including immune checkpoint inhibitors and histone deacetylase inhibitor, have been shown to exhibit promising efficacy. Tislelizumab is an anti-human programmed death receptor-1 (PD-1) monoclonal IgG4 antibody that is being developed by Bei Gene as an immunotherapeutic, anti-neoplastic drug. Tislelizumab has been investigated in haematological cancers and advanced solid tumours, leading to its approval in December 2019 in China for patients with relapsed or refractory classical Hodgkin's lymphoma after at least secondline chemotherapy [18].The registration of tislelizumab for other indications is currently underway. Chidamide (CS055, HBI-8000),a novel oral benzamide class of subtype-selective inhibitor of HDAC 1,2,3 and10,inhibited cell proliferation and interfered withPI3KAkt-mTOR and MAPK signaling. A prospective phase II trial in China illustrated that chidamide monotherapy was effective in 15 patients (detailed disease stages not reported) with relapsed/refractory extranodal NK/T-cell lymphoma [19]. In our case the patient has already received the therapy of anti-PD1 antibody with Chidamide for more than one year, showing that this treatment is effective and safe. As randomized trials comparing different regimens have not yet been conducted and standard therapy has not yet been established for these patients, treatment should be individualized based on patient, tolerance and comorbidities.

\section{Conclusion}

At this moment, there is no recommended treatment for ENKL complicated with HPS because of the extreme rarity of this entity. It was equally important to treat primary diseases as well as treating HPS. L-asparaginase-containing regimens are the cornerstone for treating ENKL. In this modern ENKL treatment era, newer agents are being investigated for treating ENKL and prospective multicenter trials need to be performed to establish an optimal treatment for this rare and dismal disease.

\section{Funding}

There was no funding support of this work.

\section{Disclosure}

The authors declare that there is no conflict of interest.

\section{References}

1. Cai Q Cai J, Fang Y, Young KH (2019) Epstein-Barr Virus-Positive Natural Killer/T-Cell Lymphoma. FRONT ONCOL 9: 386.

2. Suzuki R, Takeuchi K, Ohshima K, Nakamura S (2008) Extranodal NK/Tcell lymphoma: diagnosis and treatment cues. HEMATOL ONCOL 26(2): 66-72.

3. Chim CS, Ooi GC, Shek TW, Liang R, Kwong YL (1999) Lethal midline granuloma revisited: nasal T/Natural-killer cell lymphoma. J CLIN ONCOL 17(4): 1322-1325.

4. Harabuchi Y, Takahara M, Kishibe K, Moriai S, Nagato T, et al. (2009) Nasal natural killer (NK)/T-cell lymphoma: clinical, histological, virological, and genetic features. INT J CLIN ONCOL 14(3): 181-190.

5. Cheung MM, Chan JK, Wong KF (2003) Natural killer cell neoplasms: a distinctive group of highly aggressive lymphomas/leukemias. SEMIN HEMATOL 40(3): 221-232.

6. Gill H, Liang RHS, Tse E (2010) Extranodal natural-killer/t-cell lymphoma, nasal type. Advances in hematology 2010: 627401.

7. Kwong YL (2011) The diagnosis and management of extranodal NK/Tcell lymphoma, nasal-type and aggressive NK-cell leukemia. J Clin Exp Hematop 51(1): 21-28.

8. Jia J, Song Y, Lin N, Liu W, Ping L, et al. (2016) Clinical features and survival of extranodal natural killer/T cell lymphoma with and without hemophagocytic syndrome. ANN HEMATOL 95(12): 2023-2031.

9. Yamaguchi M, Suzuki R, Oguchi M (2018) Advances in the treatment of extranodal NK/T-cell lymphoma, nasal type. BLOOD 131(23): 25282540 .

10. Han L, Li L, Wu J, Li X, Zhang L, et al. (2014) Clinical features and treatment of natural killer/T cell lymphoma associated with hemophagocytic syndrome: comparison with another T cell lymphoma associated with hemophagocytic syndrome. Leuk Lymphoma 55(9): 2048-2055.

11. Chang Y, Cui M, Fu X, Han L, Zhang L, et al. (2018) Lymphoma associated hemophagocytic syndrome: A single-center retrospective study. ONCOL LETT 16(1): 1275-1284.

12. Fei W, Xiaohong W, Hong Z, Bei H (2015) Pulmonary Extranodal Natural Killer/T-Cell Lymphoma (Nasal Type): A Case Report and Radiological Image Review. Medicine (Baltimore) 94(38): e1527.

13. Yiu CR, Kao YH, Phipps C, Tan D (2011) Positron emission tomography findings in patients with lymphoma-associated haemophagocytic syndrome. Singapore Med J 52(7): e156-e159.

14. Au WY, Weisenburger DD, Intragumtornchai T, Nakamura S, Kim WS, et al. (2009) Clinical differences between nasal and extranasal natural killer/T-cell lymphoma: a study of 136 cases from the International Peripheral T-Cell Lymphoma Project. BLOOD 113(17): 3931-3937.

15. Yamaguchi M, Kita K, Miwa H, Nishii K, Oka K, et al. (1995) Frequent expression of P-glycoprotein/MDR1 by nasal T-cell lymphoma cells. Cancer 76(11): 2351-2356.

16. Horwitz SM, Ansell SM, Ai WZ, Barnes J, Barta SK, et al. (2018) NCCN Guidelines Insights: T-Cell Lymphomas, Version 2.2018. J Natl Compr Canc Netw 16(2): 123-135.

17. Wang JH, Wang H, Wang YJ, Xia ZJ, Huang HQ, et al. (2016) Analysis of the efficacy and safety of a combined gemcitabine, oxaliplatin and pegaspargase regimen for NK/T-cell lymphoma. Oncotarget $7(23)$ : 35412-3522.

18. Lee A, Keam SJ (2020) Tislelizumab: First Approval. DRUGS 80(6): 617624.

19. Yan G, Huang H, Li P, Bing B, Xiaoxiao W, et al. (2017) Chidamide ,Oral Subtype-Selective Histone Deacetylase Inhibitor (HDACI) Monotherapy Was Effective on the Patients with Relapsed or Refractory Extranodal Natural Killer (NK)/T-Cell Lymphoma. BLOOD 130(1) :2797. 
ISSN: 2574-1241

DOI: 10.26717/BJSTR.2021.38.006202

Changran Zhang. Biomed J Sci \& Tech Res

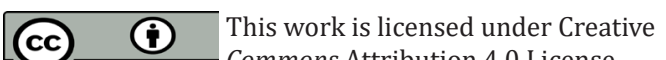

Commons Attribution 4.0 License

Submission Link: https://biomedres.us/submit-manuscript.php

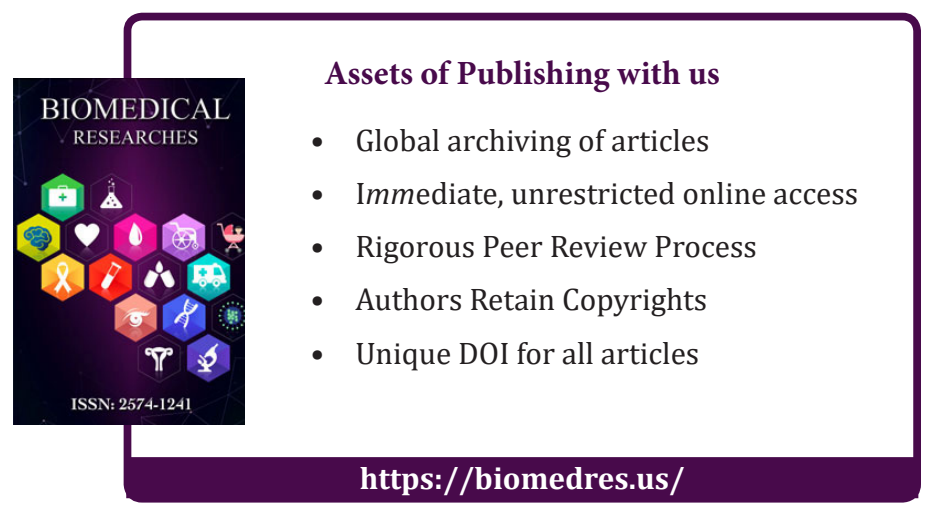

